\title{
Development of selective tau and MAO-B PET tracers through compound optimization of ${ }^{18} \mathrm{~F}$-THK5351
}

\author{
Ryuchi Harada ${ }^{1,2}$, Nobuyuki Okamura ${ }^{3,4}$, Shozo Furumoto ${ }^{4}$, Hiroyuki Arai ${ }^{2}$, Yukitsuka Kudo ${ }^{2}$, \\ Kazuhiko Yanai ${ }^{1,4}$
}

${ }^{I}$ Pharmacology, Tohoku University Graduate School of Medicine, Japan, ${ }^{2}$ Institute of Development, Aging and Cancer, Tohoku University, Japan, ${ }^{3}$ Pharmacology, Tohoku Medical and Pharmaceutical University, School of Medicine, Japan, ${ }^{4}$ Cyclotron and Radioisotope Center, Tohoku University, Japan

Background/Aims: Validation studies have shown that ${ }^{18} \mathrm{~F}$-THK5351 PET signal contribute the binding to monoamine oxidase $\mathrm{B}(\mathrm{MAO}-\mathrm{B})$ and correlates with tau and astrogliosis in neurodegenerative diseases. The aim of study was to develop selective tau and MAO-B PET tracers through compound optimization from ${ }^{18} \mathrm{~F}$-THK5351 to visualize tau pathology and astroglisis, respectively.

Methods: In vitro competitive binding assay against recombinant $\mathrm{MAO}-\mathrm{B}, \mathrm{MAO}-\mathrm{A}$, and tau aggregates in $\mathrm{AD}$ brain homogenates was performed to evaluate binding affinity of test compounds. In vitro autoradiography in frozen human brain tissues was performed using ${ }^{18} \mathrm{~F}$-labeled compounds to evaluate selective binding to each target.

Results: In vitro competitive binding assays demonstrated lower binding affinity of several quinoline derivatives against MAO-B (Ki > $100 \mathrm{nM}$ ) than THK5351. On the other hand, we identified several quinoline derivatives that possess higher affinity against MAO-B than THK5351, but little binding to MAO-A and tau aggregates. Furthremore, in vitro autoradiography of $\mathrm{AD}$ brain sections showed the selective binding ability of these compounds to tau protein deposits or MAO-B.

Conclusions: Compound optimization resulted in successful improvement of binding selectivity of quinoline derivatives as tau and MAO-B selective PET tracers, respectively. Selective imaging of tau and MAO-B would be promising markers for monitoring neurodegeneration and neuroinflammatory changes. 